\title{
New Method to Improve The Gain Ripple in Single and Double Stage Double Pass Erbium Doped Fiber Amplifier in Multichannel System
}

\author{
Aied K. Mohammed, PhD \\ Department of Electrical Engineering, \\ University of Technology
}

\author{
Noor Yahya Jawad \\ Department of Electrical Engineering, \\ University of Technology
}

\begin{abstract}
In this paper two configurations single and double stage with double pass technique are used with new flatting technique. The "Optisystem version 13.0" software package is used for simulation process. By using single stage EDFA with double pass technique the gain ripple enhanced from $34.56 \pm 2.8$ to $35.21 \pm 0.43 \mathrm{~dB}$ with using flatting technique between the first and second passes, but it has high noise figure $5.98 \pm 1.12 \mathrm{~dB}$. The gain in the double stage EDFA with double pass technique is improved from $33.67 \pm 3$ to $34.60 \pm 0.56 \mathrm{~dB}$ with using mid stage flatting technique and the noise figure of it is $3.94 \pm 0.3 \mathrm{~dB}$.
\end{abstract}

\section{Keywords}

EDFA, single stage, double stage, double pass, gain flatting, gain ripple

\section{INTRODUCTION}

A communication system transmits information from one place to another, whether separated by a few kilometers or by transoceanic distances. Information is often carried by an electromagnetic carrier wave whose frequency can vary from a few megahertz to several hundred terahertz. Optical communication systems use high carrier frequencies $(\sim 100$ $\mathrm{THz}$ ) in the visible or near-infrared region of the electromagnetic spectrum. [1].

In any transmission medium the signal is attenuated or suffers loss, and is subject to degradations in transmitted signals, for this reason the amplifier should be used to compensated for the loses[2].

Attenuation in optical fiber leads to a reduction of the signal power as the signal propagates over some distance. The maximum distance between the transmitter and the receiver (or the distance between amplifiers) depends more heavily on attenuation [3]. Signal attenuation is defined as the ratio of optical input power $\left(\mathrm{P}_{\mathrm{i}}\right)$ to the optical output power $\left(\mathrm{P}_{\mathrm{o}}\right)$. Optical input power is the power injected into the fiber from an optical source. Optical output power is the power received at the fiber end. The following equation defines signal attenuation per a unit of length

Attenuation $(\alpha)=\left(\frac{10}{L}\right) \log _{10}\left(\frac{P_{i}}{P_{o}}\right)$

Where, length $(\mathrm{L})$ is expressed in kilometers. Therefore, the unit of attenuation is decibels $/$ kilometer $(\mathrm{dB} / \mathrm{km})$. Attenuation in an optical fiber is caused by absorption, scattering, and bending losses [1].

Optical amplifiers, as their name implies, operate solely in the optical domain with no inter-conversion of photons to electrons [2]. The signal is optically amplified in strength by several orders of magnitude without being limited by any electronic bandwidth. The shift from regenerators to amplifiers thus permits a dramatic increase in capacity of the transmission system. Optical amplifiers play an important role in increasing the capacity of optical communication systems using such amplifiers [4]. Optical amplifiers have been essential elements for high capacity, long-lifespan and multiple connection of optical for communication network applications [5].

\section{ERBIUM DOPED FIBER AMPLIFIER (EDFA)}

An important class of lumped optical amplifiers makes use of rare-earth elements as a gain medium by doping the fiber core during the manufacturing process. Although doped-fiber amplifiers were studied as early as 1964 , their use became practical only 25 years later, after their fabrication techniques were perfected. In such amplifiers, properties such as the operating wavelength and the gain bandwidth are determined by the dopants while silica plays the role of a host medium [1]. The general components of the doped fiber amplifier are, doping fiber, pumping source, isolator, and coupler, as shown in Figure (1).

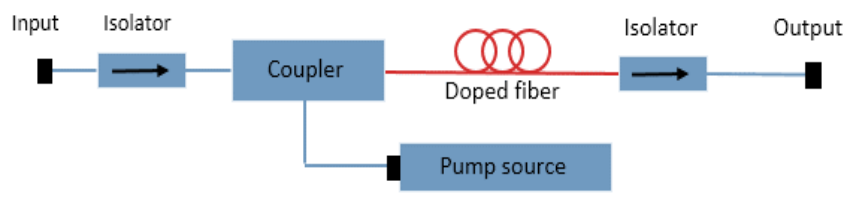

Fig 1: General components of fiber amplifier

The Erbium-Doped Fiber Amplifier was the first optical amplifier widely used in optical communications systems, it has become a key component in many optical networks because it provides efficient, low-noise amplification of light in the optical fiber low-loss telecommunications window near $1550 \mathrm{~nm}[6,7]$. An EDFA has several advantages as follows:

- The power conversion efficiency from pump to signal is high.

- Directly and simultaneously amplify a wide wavelength region (in the region of $1550 \mathrm{~nm}$ ) with a relatively flat gain $(>20 \mathrm{~dB})$, which is suitable for WDM systems.

- $\quad$ Large dynamic range (> $80 \mathrm{~nm}$ ).

- Low noise figure.

- Polarization independent (thus reducing coupling loss to transmission fiber).

- Suitable for long-haul applications. 
- $\quad$ Modified EDFAs can also operate in the L-band.[8]

There is disadvantage in EDFA as following: EDFAs exhibit amplified spontaneous light emission (ASE). That is, even if no incoming signal is present, there is always some output signal as a result of some excited ions in the fiber, this output is termed spontaneous noise.

\section{PASS TECHNIQUE}

When the signal travels in gain medium at one time as shown in Figure (1), this configuration is called single pass. When the signal travels through the gain fiber twice goings and comings, this amplifier configuration is called the doublepass, as shown in Figure (2) [9].

OC: Optical Circulator

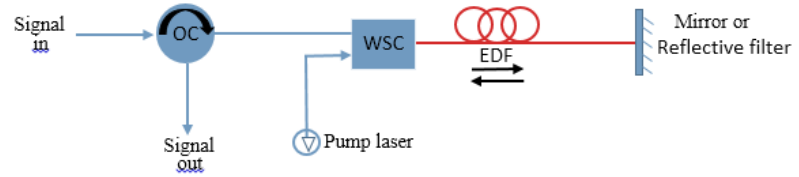

Fig 2: Double pass configuration

The circulator component is a passive multifiber junction where an incoming signal is routed to another fiber in a clockwise direction [10]. The signal reflector is used to reflect the signal to active medium, there are several types of reflector such as mirror, reflective filter, and fiber brag grating (FBG). Because of the FBG band reflector is narrow, it used to suppress the ASE noise.

When the reflected signal is back to EDF, it will be amplified for the second time. The double pass has several advantages over single pass, it achieves high-power conversion efficiency, high gain, and high output power $[9,6]$.

\section{DESIGN OF SINGLE AND DOUBLE STAGE EDFA WITH DOUBLE PASS TECHNIQUE}

In this section different configurations will be design to get best performance.

\subsection{Single stage EDFA with double pass technique}

Figure (3) shows such design in which 32 channels are used with the channel spacing of $120 \mathrm{GHz}$ and bandwidth of 10GHz. The EDFA has the following parameters

Table 1: EDFA parameters

\begin{tabular}{|c|c|}
\hline EDFA's parameter & value \\
\hline Active fiber length & $4.2 \mathrm{~m}$ \\
\hline Er concentration & $10 e^{+24} \mathrm{~m}^{-3}$ \\
\hline Fiber NA & $0.24 \mathrm{NA}$. \\
\hline Pump power & $250 \mathrm{mw}$ \\
\hline
\end{tabular}

These parameters were chosen according to experience and many tests were made to get better enhancement results. The reflective filter bidirectional (like mirror) is used to reflect the signals to EDF, this filter has center wavelength of $1545 \mathrm{~nm}$, bandwidth $32 \mathrm{~nm}$, and reflectivity of $99 \%$.

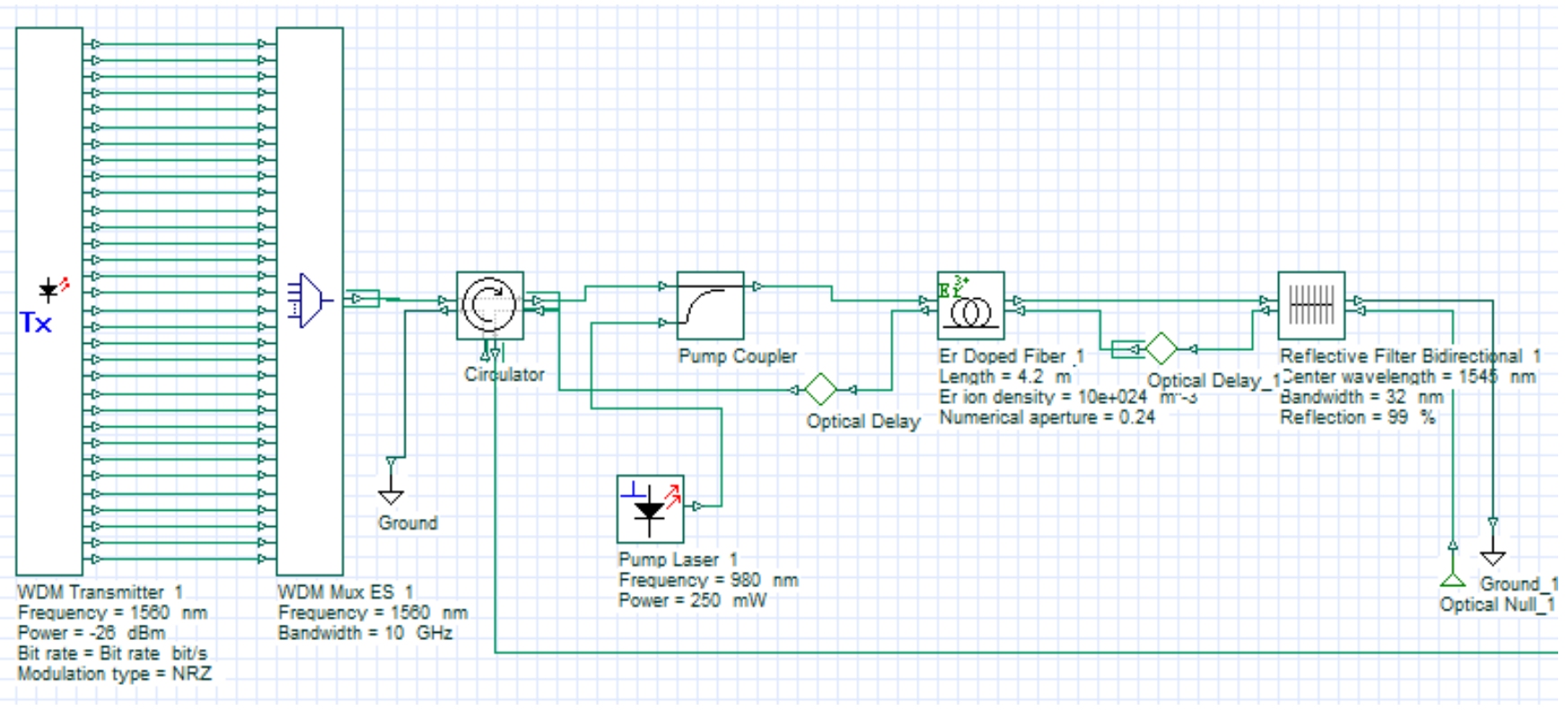

Fig 3: Single stage double pass configuration for C-band

To improve the gain flatness a flatting technique is used between the EDF and reflective filter bidirectional, this technique constructed from Gaussian filter and X-coupler, the two UFBG are used after circulator for more flatting gain, as shown in Figure (4) 


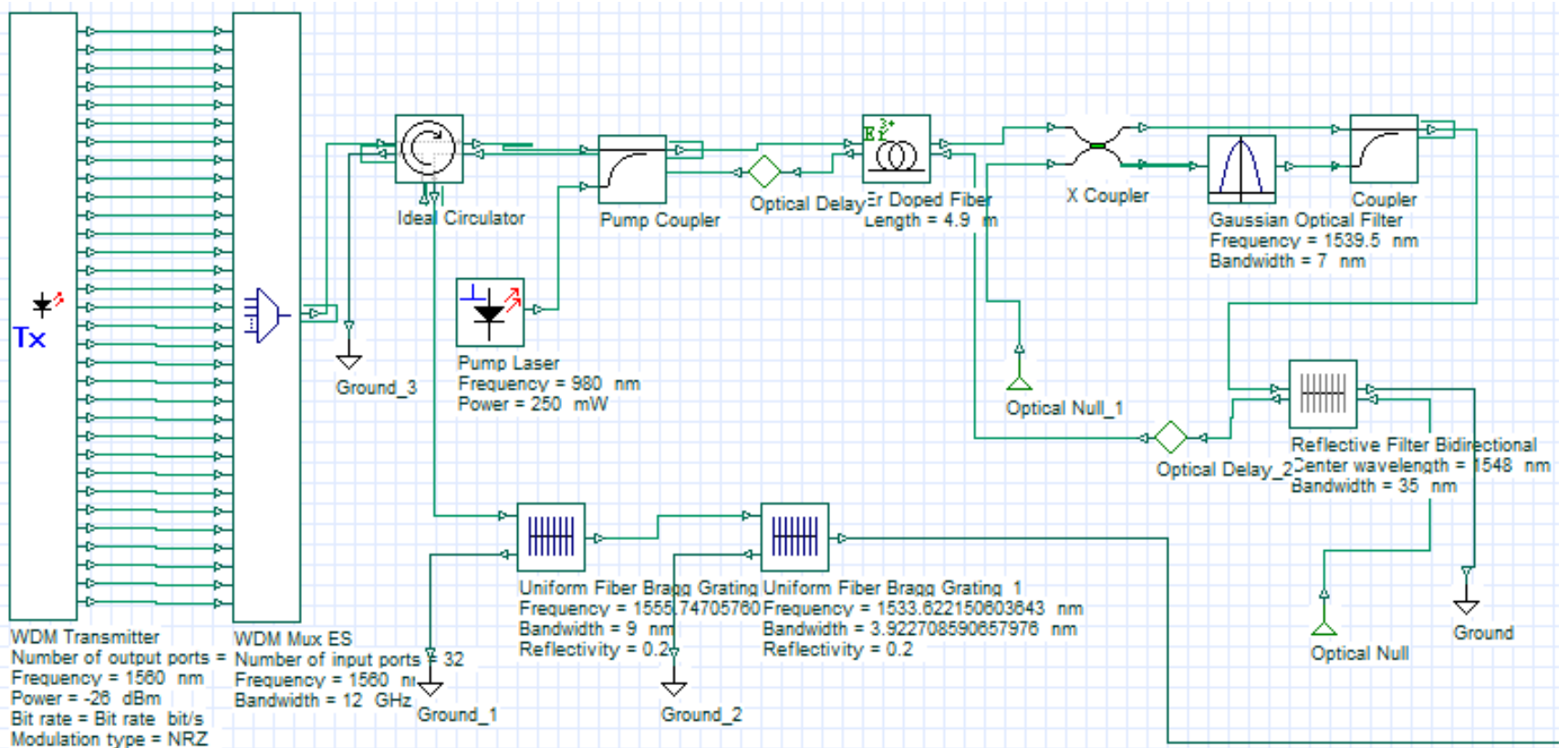

Fig 4: Single stage double pass configuration to improve gain flatness

The purpose of this design is to get flat gain over wide range of frequencies. The Gaussian band pass filter (BPF) is used for flatting a certain region of C-band as shown in Figure (4). This process is done by dividing the signal into two branches by using X-coupler with 0.57 coupling coefficient.

The second branch connected to the BPF which has center wavelength of $1539.5 \mathrm{~nm}$ with bandwidth of $7 \mathrm{~nm}$, after the $\mathrm{BPF}$, the coupler is used to combine these branches as shown in Figure (4).

After the circulator the signal passes through two Uniform Fiber Bragg Gratings (UFBG) with bandwidths of 9nm and $3.53 \mathrm{~nm}$, the center wavelengths of $1555.74 \mathrm{~nm}$ and $1533.62 \mathrm{~nm}$, and reflectivity of $20 \%$ and $20 \%$ respectively.

\subsection{Double stage EDFA with double pass technique}

Figure (5) shows such design in which 32 channels are used with the channel spacing of $120 \mathrm{GHz}$ and bandwidth of 10GHz. In this design two EDFAs are used and they have the following parameters:

Table 2: Parameters of two EDFAs

\begin{tabular}{|c|c|c|}
\hline parameters & First stage & Second stage \\
\hline EDF length & $1 \mathrm{~m}$ & $3.7 \mathrm{~m}$ \\
\hline Er concentration & $10 \mathrm{e}^{24} \mathrm{~m}^{-1}$ & $10 \mathrm{e}^{24} \mathrm{~m}^{-1}$ \\
\hline Pumping power & $200 \mathrm{mw}$ & $50 \mathrm{mw}$ \\
\hline NA & 0.24 & 0.24 \\
\hline
\end{tabular}

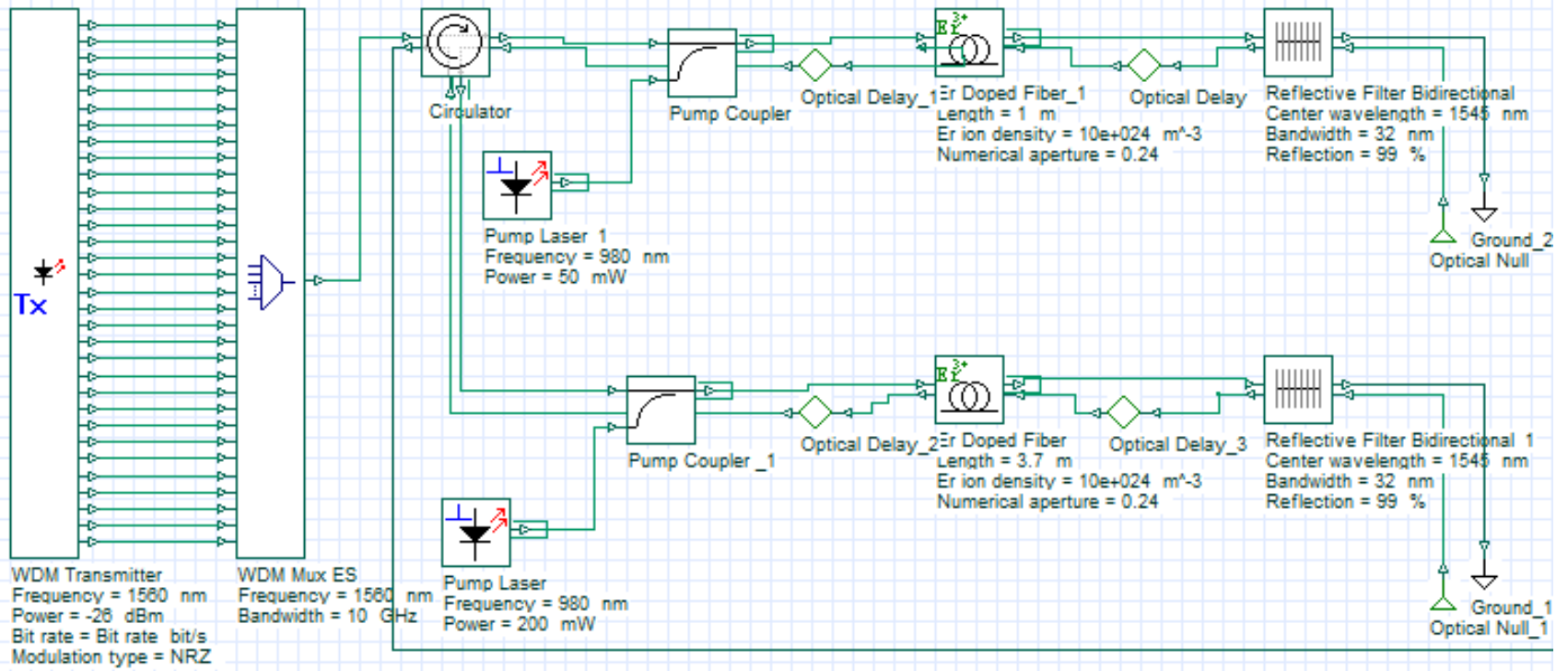

Fig 5: Double stage double pass design at C-band

To get more flatting in gain over full C-band a midstage is used between two stages of EDFA of parallel configuration. As shown in Figure (6), this midstage consists of X-coupler with coupling coefficient of 0.62 , and optical Gaussian filter which has a center wavelength of $1539.5 \mathrm{~nm}$ with bandwidth $7 \mathrm{~nm}$. 


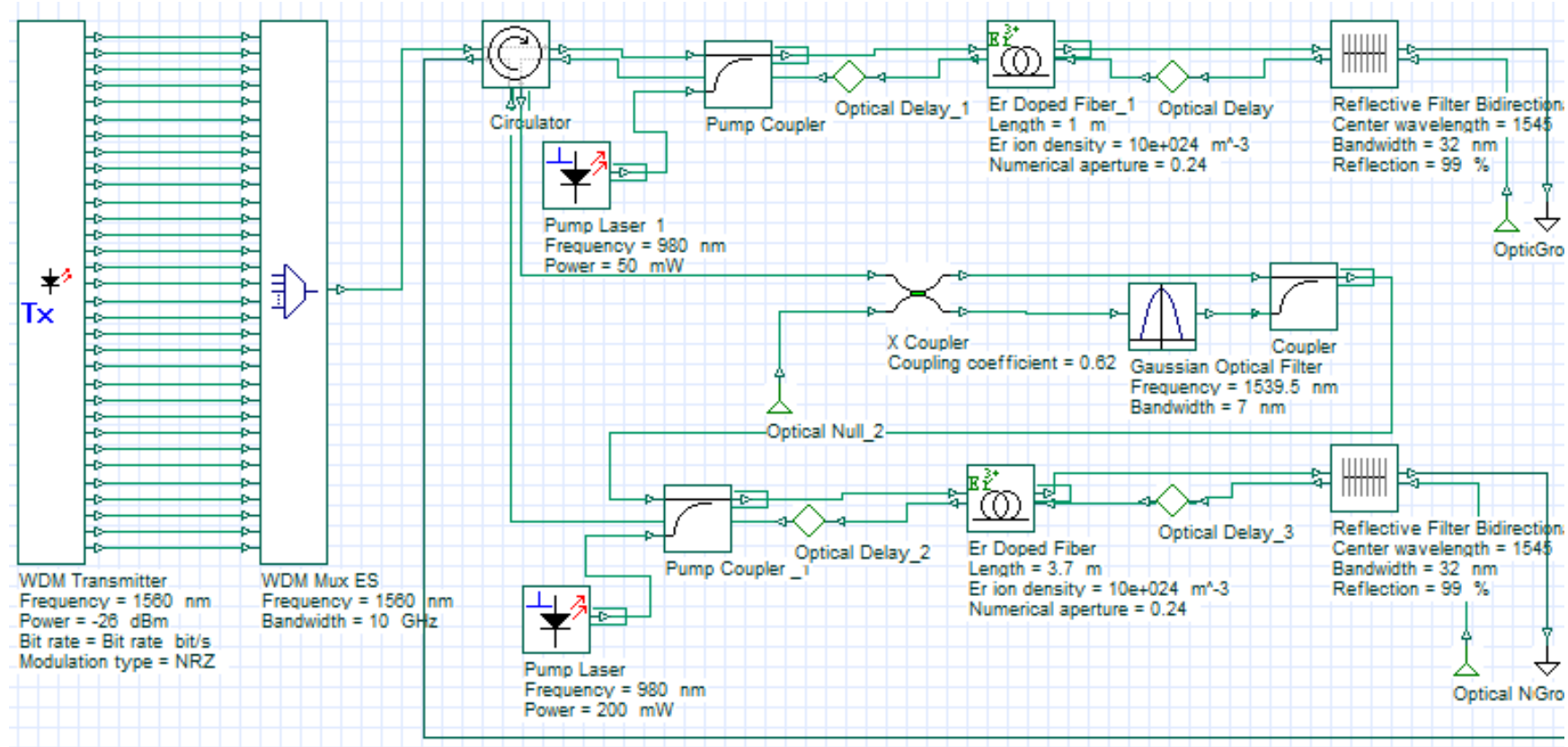

Fig 6: Double stage double pass at $\mathrm{C}$-band with midstage

\section{SIMULATION RESULTS}

This section show the simulation results of the designs in previous section.

\subsection{Single stage EDFA with double pass technique}

The single stage double pass EDFA design, shown in Figure (3), has been simulated, the gain for first pass, second pass, and overall are shown in Figure (7)

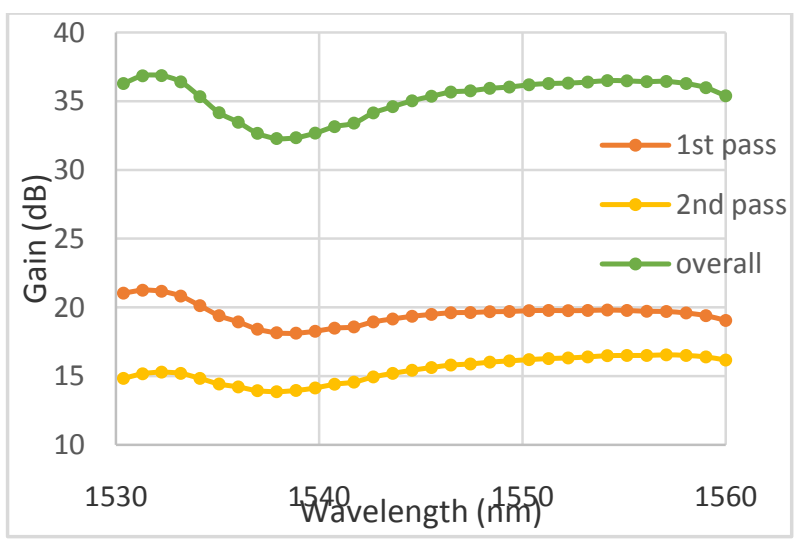

Fig 7: Gain for $1^{\text {st }}, 2^{\text {nd }}$, and overall pass for $\mathrm{C}$ - band

The overall gain is high due to twice pass of signals through the active fiber, and this technique reduced the length of EDF to a half.

The overall noise of single stage double pass is high as shown in Figure (8), due to double passing that make the backward ASE very strong. The overall noise figure is same as first pass noise figure, because the first and second pass are considered two stages and the noise figure of first stage has more effect on total noise figure than other stages.

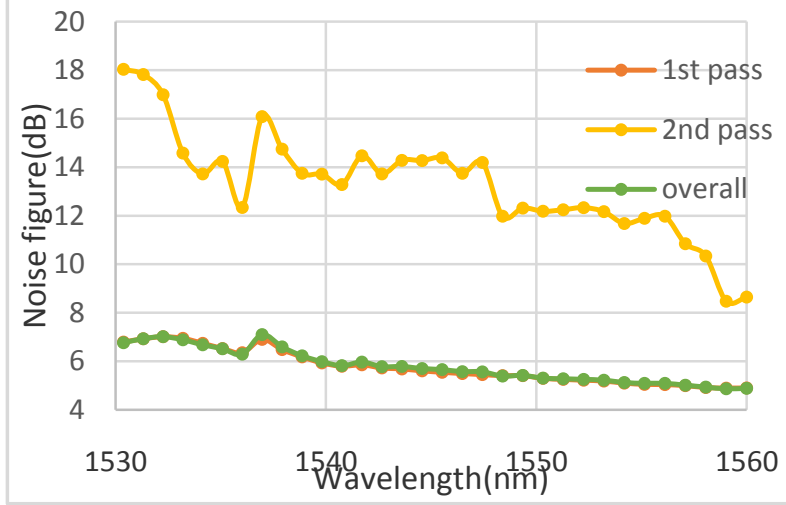

Fig 8: Noise figure for $1^{\text {st }}, 2^{\text {nd }}$, and overall pass for Cband

By using the filter improvement between first and second passes and two UFBG after second pass as show in Figure (4), the gain ripple is enhanced to $0.87 \mathrm{~dB}$, while without such addition it was $4.54 \mathrm{~dB}$. Figure (9) shows the gain variation at $\mathrm{C}$-band for different design configurations.

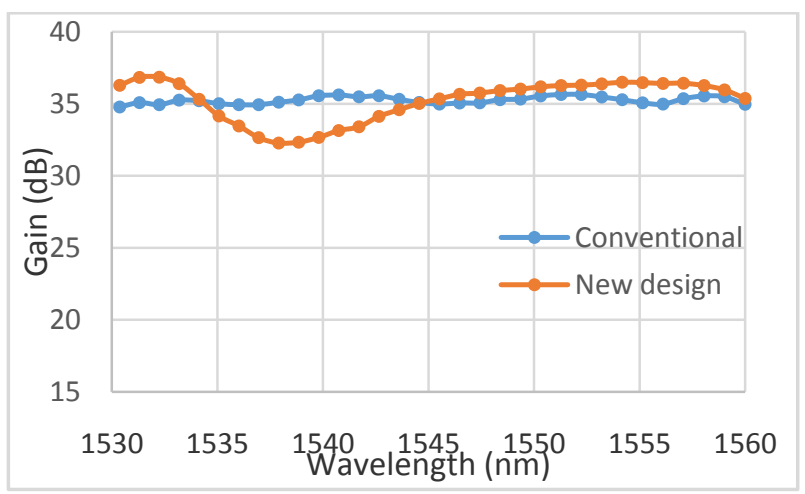

Fig 9: Gain variation at different configurations of single stage double pass at $\mathrm{C}$-band

The noise figure for two configurations is also studied and the simulation results are shown in Figure (10) 


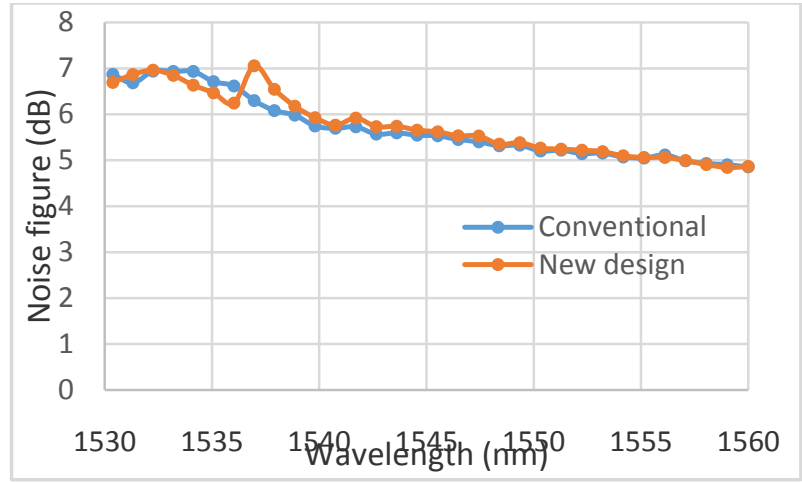

Fig 10: Variation of noise figure at different design configurations.

\subsection{Double stage EDFA with double pass technique}

The design of double stage with double pass technique showed in Figures (5) and (6) has been simulated and Figure (11) shows the gain variation for different designs.

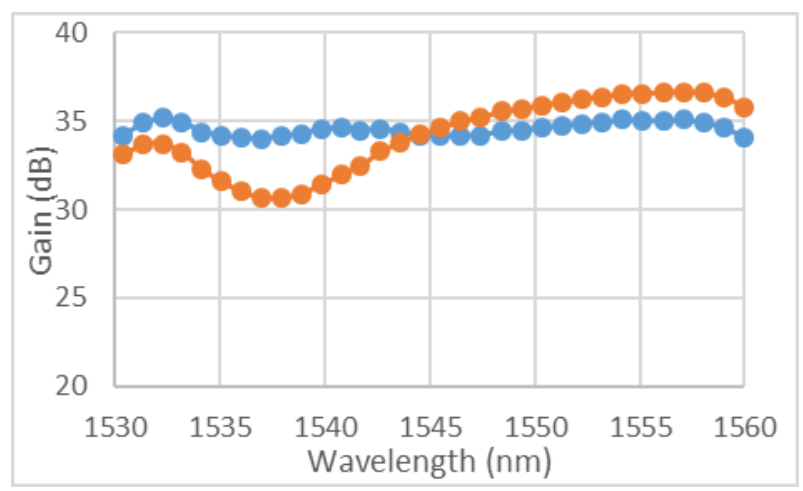

Fig 11: Gain variation of double stage double pass with and without improvement

The gain ripple for conventional design (without improvement) is $6 \mathrm{~dB}$, and after using Gaussian filter the ripple is reduced to $1.18 \mathrm{~dB}$.

The noise figure for two configurations is also studied and the simulation results are shown in Figure (12).

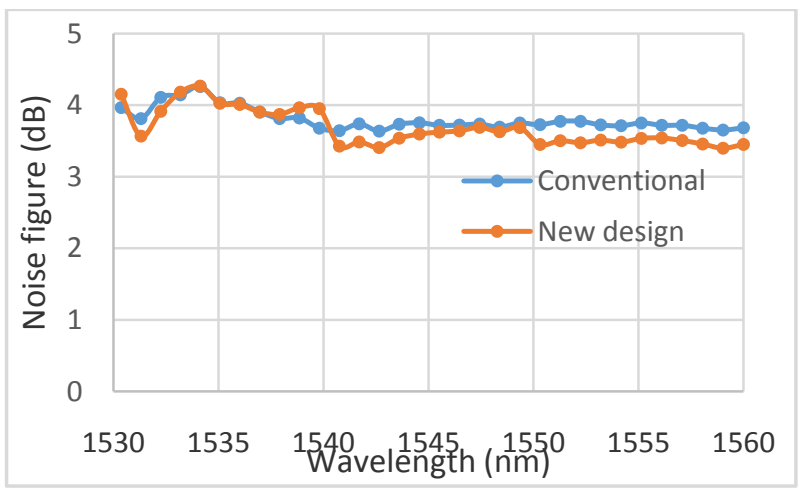

Fig 12: Noise figure variation of parallel double stage double pass with and without improvement

The single stage double pass design in previous section provides good gain whereas the noise is high to overcome this problem in double pass technique, the double stage double pass is used.

The cause that makes the noise of double stage double pass low is due to use two separated stages, always the noise figure is mainly dependent on the first stage, the first stage is constructed from short EDF and small pumping power as shown in Figure (5) to achieve small ASE forward and backward as possible that leads to small noise figure.

\section{CONCLOSION}

The new flatting method give good gain flatting for wide range of frequencies. Using this flatting method between the first and second pass in single stage EDFA the gain ripple enhanced to $\pm 0.43 \mathrm{~dB}$ along $\mathrm{C}$-band, but the single stage EDFA with double pass has high noise figure due to backward ASE. Using the flatting method for the double stage EDFA with double pass technique is give $0.56 \mathrm{~dB}$ gain flatting along $\mathrm{C}$-band with low noise figure, that make it is better than single stage with double passing technique. For future it can be used this flatting technique for L- band optical amplifier design.

\section{REFERENCES}

[1] G. P. Agrawal, "Fiber-Optic Communication Systems", John Wiley \& Sons, Fourth Edition, 2002.

[2] C. F. Lam, "Passive Optical Networks: Principles and Practice" Academic Press, 2007.

[3] K. M. Sivalingam, and S. Subramaniam, "Optical WDM Networks: Principles and Practice", Springer Science \& Business Media, 2006.

[4] Lekshmi.S.R, Sindhu.N, "Spectral Response of FWM in EDFA for Long-haul Optical Communication", International Journal of Innovative Research in Computer and Communication Engineering, Vol. 2, Issue 10, pp. 6091-6095, October 2014.

[5] M.A.Othman , M.M. Ismail , M.H.Misran, M.A.M.Said and H.A.Sulaiman, "Erbium Doped Fiber Amplifier (EDFA) for C-Band Optical Communication System", International Journal of Engineering \& Technology IJET-IJENS, Vol 12, No. 4, pp. 48-50, 2012.

[6] M. J. F. Digonnet, "Rare Earth Doped Fiber Lasers and Amplifiers",Marcel Dekker, 2001.

[7] J. Zyskind, A. Srivastava, "Optically Amplified WDM Networks”, Elsevier, 2011.

[8] S. v. Kartalopoulos," Introduction to DWDM technology” A John Wiley \& Sons, 2000.

[9] P. Urquhart, “Advances in Optical Amplifiers”, InTech, 2011. 